\title{
Assessment of Cardiovascular Effects of Auricular Sympathetic Point Using Acceleration Photoplethysmogram Indices in Healthy Subjects
}

\author{
Eduardo Becerril-Borja, Moisés S. Castañeda-Ramírez \& José F. Rivas-Vilchis \\ Especialización en Acupuntura y Fitoterapia, División de Ciencias Biológicas y de la Salud, Unidad Iztapalapa, Universidad \\ Autónoma Metropolitana. Email: jfrv@xanum.uam.mx*
}

DOI: http://doi.org/10.38177/ajast.2021.5412

Copyright: (ㅇ 2021 Eduardo Becerril-Borja et al. This is an open access article distributed under the terms of the Creative Commons Attribution License, which permits unrestricted use, distribution, and reproduction in any medium, provided the original author and source are credited.

Background: Sympathetic auricular acupoint has been used in ear acupuncture to treat hypertension and other cardiovascular diseases. However, no experimental studies fully evaluate its cardiovascular action. The indices resulting from the acceleration photoplethysmogram (APG) are used to examine cardiovascular physiology, arterial aging, and the action of vasopressor or inotropic drugs. This work was aimed to examine whether acupuncture at the sympathetic auricular point modifies the indices obtained from the APG.

Materials and methods: The indices of the second derivative of the photoplethysmogram $\left(\mathrm{d}^{2} \mathrm{PPG} / \mathrm{dt}^{2}\right)$ or acceleration photoplethysmogram (APG) namely aging index or APG-AI, APG-b/a, and APG-d/a, were calculated in 9 healthy subjects ( 5 women) aged $34.7 \pm 6.0$ years (mean \pm SD). A 5 min photoplethysmographic record was obtained. The sympathetic point of the right ear was stimulated by manual acupuncture for $60 \mathrm{~s}$ (min 3 ). Pooled APG indices of all subjects were compared at baseline, acupuncture, and after acupuncture, minutes 1-2, 3, and 4-5, respectively. A p-value $<0.05$ was considered significant.

Results: Acupuncture at the sympathetic point of the right ear produced a significant increase in the APG-b/a index and a significant decrease in the APG-d/a index in both cases during the period of acupuncture stimulation.

Conclusions: Applying the sympathetic auriculopuncture point caused significant changes in the APG-b/a and APG-d/a indices. These changes are similar to those that originate with the administration of vasoconstrictors such as angiotensin II. The changes suggest variations elicited through auriculopuncture in the anterograde and retrograde circulatory components of the arterial circulation.

Keywords: Sympathetic auricular acupoint, Auriculopuncture, Photoplethysmography, Acceleration photoplethysmogram, Digital volume pulse Second derivative of digital volume pulse indices.

\section{Introduction}

Auricular acupuncture assumes that a representation of the human body is found in the ear, and points of the ear correspond to each part of the human anatomy [1],[2]. Several articles show that auricular acupuncture can help control blood pressure. [3],[4]. Photoplethysmography (PPG), an optical measurement technique, is used to detect blood volume changes in the microvascular bed of tissue [5]. PPG has been used to evaluate arterial compliance concerning changes in wave amplitude [6],[7] and to assess hypertension [8].

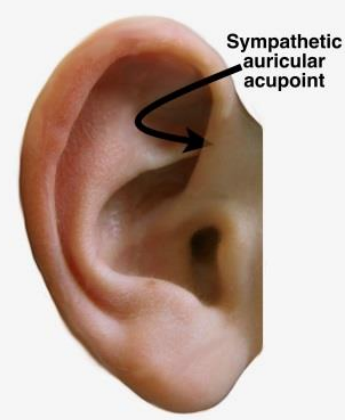

Fig.1. Sympathetic auricular acupoint

The APG allows more accurate recognition of the inflection points in the original plethysmographic wave. [9]. The APG upgrades accurate recognition of the circulation phase, and it is easier to interpret than the original PPG [10]. 
Epidemiological studies show that the information extracted from the APG reflects the elasticity of the aorta, the peripheral vessels and that it is precisely related to age and other risk factors for atherosclerotic vascular disease. [11],[12]. The study aimed to investigate the acute effects of manual needling in sympathetic auricular acupoint (Fig.1) on APG indices in healthy subjects.

\section{Material And Methods}

Study design: A single-center, non-randomized, experimental trial was conducted to verify the kinetics of manual acupuncture at the right ear sympathetic acupoint on APG indices in healthy subjects.

Participants: The study group consisted of 9 (5 women) healthy subjects with a mean age ( \pm SD) of $34.7 \pm 6.0$ years. All subjects were normotensive (office blood pressure $<140 / 90 \mathrm{mmHg}$ ), none had total serum cholesterol $>200 \mathrm{mg} / \mathrm{dl}$ or glucose $>100 \mathrm{mg} / \mathrm{dl}$ at the time of the study. No subjects participating in the study had heart or kidney disease or were taking any medications. None of the participants had cardiovascular or kidney disease.

Ethical aspects: The Ethics Committee of the Biological and Health Sciences Division of the Autonomous Metropolitan University of Iztapalapa (2015.6.03.06) approved the trial. All participants gave informed consent.

Instrumentation and records: The second derivative of the PPG wave record was obtained using Origin, Scientific Graphing and Analysis software, v. 7.5 (Microcal Software, Inc., Northampton, MA); with this trace, it was possible to obtain the inflection points of the indices. Typically, the $\mathrm{d}^{2} \mathrm{PPG} / \mathrm{dt}^{2}$ waveform comprises five distinct waves: a through e. Each wave measured from the baseline has positive or negative values when it is above or under the baseline, respectively [13].

PPG registration: A photoplethysmography transducer (TSD 200; BIOPAC Systems, Goleta, CA, USA) was used, which transmits infrared light at $860 \pm 90 \mathrm{~nm}$ to obtain the PPG. The transducer was placed on the index finger of the left hand of each participating subject. The frequency response of the photoplethysmograph was flat at $10 \mathrm{~Hz}$. The digital output of the photoplethysmograph was recorded through a 12-bit analog-to-digital converter with a sampling rate of 200 points per second (MP100; BIOPAC Systems, Goleta, CA, USA). The analysis platform provided by the Acknowledge software version 3.8.1 (MP100; BIOPAC Systems, Goleta, CA, USA) was used.

APG indices: The second derivative of the PPG wave $\left(\mathrm{d}^{2} \mathrm{PPG} / \mathrm{dt}^{2}\right)$ was obtained using Origin software, Scientific Graphing and Analysis Software version 7.5 (Microcal Software, Inc., Northampton, MA, USA). The d $\mathrm{d}^{2} \mathrm{PPG} / \mathrm{dt}^{2}$ or $^{2}$ APG wave typically comprises five distinct waves: a to e. The APG indices are calculated using the relative height of the positive or negative inflections. The values of wave $b$ and wave $d$ are normalized to wave $a$, then the b/a and $\mathrm{d} / \mathrm{a}$ relationships are obtained. The aging index of the second derivative (APG-AI) is defined as the quotient of the waves of the algebraic sum (b-c-d-e) normalized with the value of wave a [14]. The data of each subject corresponding to each minute were graphed, and the interpolated data of the eight subjects were averaged with the Plot2 software (Michael Wesemann, Berlin, Germany, 2019), and a kinetic curve of the effect of acupuncture was obtained. Subsequently, the curves corresponding to each variable were interpolated in a single graph.

Intervention: The right sympathetic point was chosen for its known cardiovascular effects. We use $13 \mathrm{~mm}$ long $0.22 \mathrm{~mm}$ diameter disposable stainless steel needles (Hawto, China, Beijing, China) without additional electrical or 
laser stimulation. The previous evaluation included the recording of the clinical history and symptoms. An acupuncture needle was unilaterally inserted into the sympathetic point (Fig.1) at a depth of $2 \mathrm{~mm}$ in the right ear without additional stimulation. All subjects received the same care and acupuncture treatment. The trial was blinded to the clinical investigator who calculated the APG indices. The acupuncture treatment was administered by a physician trained in conventional western medicine and traditional Chinese medicine.

Study protocol: All the subjects were studied fasting, and they abstained from consuming caffeine, alcohol, or smoking in the previous $12 \mathrm{~h}$. Measurements were made with each subject in the supine position. All records were made in the morning in a laboratory with a temperature of $23 \pm 1^{\circ} \mathrm{C}$. All subjects rested for at least 20 minutes before the start of the recordings. A TSD200 photoplethysmograph (BIOPAC Systems, Inc., Goleta, CA, USA) transmitting infrared light at $860 \mathrm{~nm} \pm 90 \mathrm{~nm}$ placed on the index finger of the left hand was used to obtain the PPG.

The digital output of the photoplethysmograph was recorded through a 12-bit analog-to-digital converter with a sampling frequency of 200 points per second with the BIOPAC Systems instrument, MP150 (BIOPAC Systems, Inc., Goleta, CA, United States), and the use of the AcqKnowledge v. 4.1 (BIOPAC Systems, Inc., Goleta, CA, United States). A continuous PPG recording of 5 min duration was obtained from each subject. After a baseline recording of $2 \mathrm{~min}$, the sympathetic point was manually stimulated in the right ear, contralateral to the side of the PPG recording. The sympathetic point of the right ear was stimulated by a manual puncture for one $\min (\min 3)$. Continuous, high-quality APF indices were calculated from beat-to-beat PPG for each subject in the baseline period (min 1 and 2), during acupuncture (min 3), and after acupuncture (minutes 4 and 5). The APG indices for each group were averaged and then tested for statistically significant differences between the two groups. Given the wide variation of the APG indices to obtain a reliable version of the peaks and their intensities, a smoothed function was obtained through the fast Fourier transform of the signal for each data set of APG indices [15].

Outcomes: The APG-b/a, APG-d/a, and APG-AI indices were calculated in the baseline period (min 1-2), during the acupuncture period $(\min 3)$, and after the acupuncture period $(\min 3-5)$.

Data analysis: Based on absolute values for the height of waves a, b, c, and d from the raw PPG, we calculate and analyze the following variables: APG-b/a, that is, the relationship between the height of wave $b$ and wave $a$; and similarly, APG-d/a; and APG-(b-c-d-e)/a, called the aging index or APG-AI [13].

Data interpolation: The records of the subjects of each group were plotted in the Plot2 software (Michael Wesemann, Berlin, Germany, 2019). The interpolated data of the nine subjects of the experimental group were averaged, and kinetic curves of the behavior of the APG indices were obtained. Baseline values were compared with those corresponding to the acupuncture stimulation period and the after acupuncture period.

Smoothing of grouped data: The interpolated data from each APG index was smoothed using fast Fourier transform in Plot2 software (Michael Wesemann, Berlin, Germany, 2019).

Statistic analysis: Student's t-test was used to compare normally distributed continuous variables. The level of statistical significance chosen was $\mathrm{p}<0.05$. All statistical analyzes were performed using the statistical package for social sciences (SPSS, version 22.0, SPSS Inc., Chicago, IL, USA). 


\section{Results}

Table 1 shows the demographic characteristics of the studied group.

Table 1. Demographic characteristics of the studied group.

\begin{tabular}{|c|c|c|}
\hline Parameter & n & Age \\
\hline Total & 9 & $34.7 \pm 6.0$ \\
\hline $\begin{array}{c}\text { Women } \\
\mathbf{n}(\%)\end{array}$ & $5(55.6 \%)$ & $33.0 \pm 6.4$ \\
\hline $\begin{array}{c}\text { Men } \\
\mathbf{n}(\%)\end{array}$ & $4(44.4 \%)$ & $36.8 \pm 5.6$ \\
\hline
\end{tabular}

*Data are presented as mean \pm standard deviation.

Fig. 2 shows a typical raw recording of the digital pulse photoplethysmogram of 5 minutes.

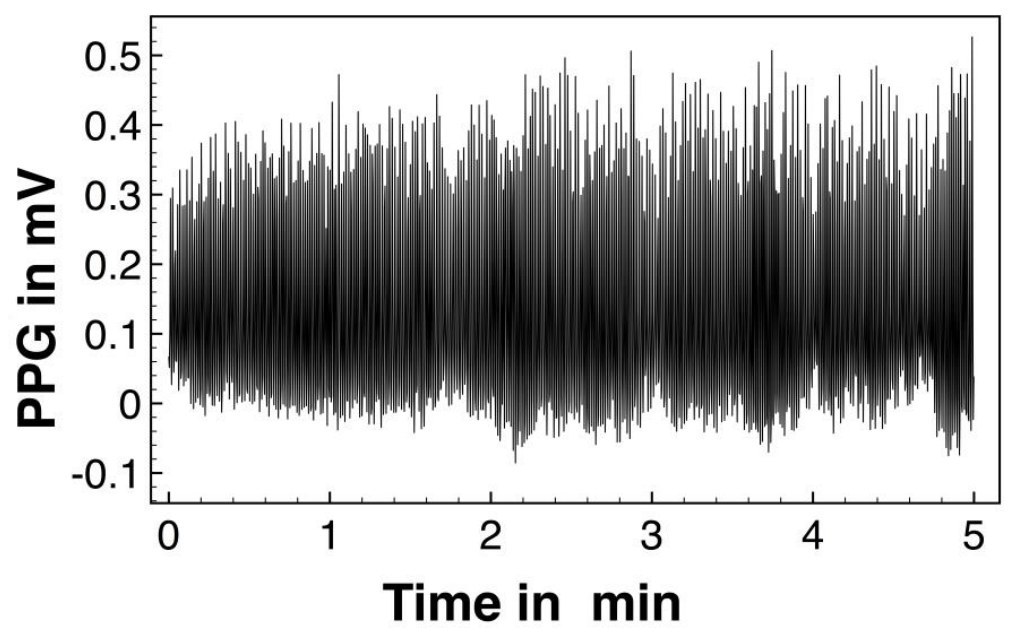

Fig.2. Characteristic record of the digital photoplethysmogram (PPG)

Acceleration photoplethysmogram (APG): The second derivative of the photoplethysmogram (SDPPG) or acceleration photoplethysmogram (APG) is shown in Fig.3.

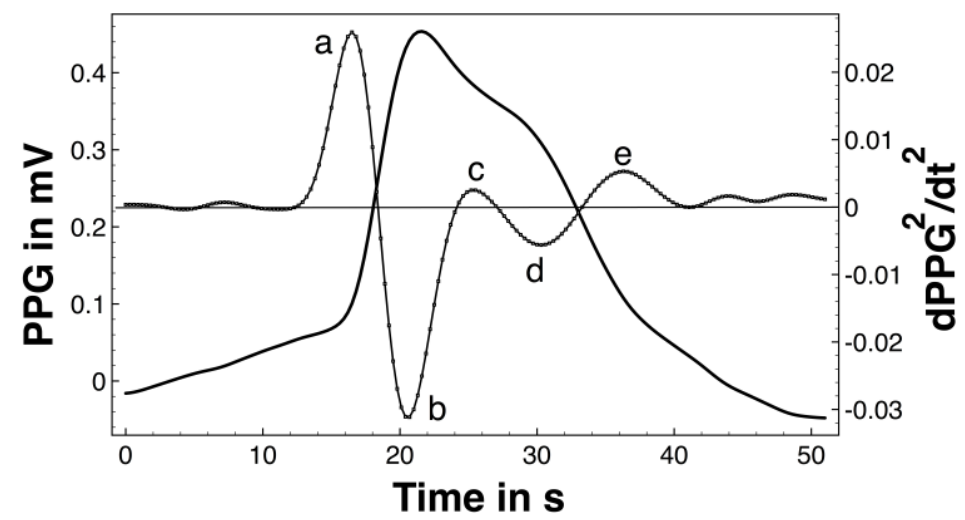

Fig.3. The second derivative of the photoplethysmogram $\left(\mathrm{d}^{2} \mathrm{PPG} / \mathrm{dt}^{2}\right)$ or acceleration photoplethysmogram (APG) with its five primary waves: a, c, d positives; $b$, and d negatives 
The $\mathrm{d}^{2} \mathrm{PPG} / \mathrm{dt}^{2}$ or APG characteristically shows five primary waves named a- e [13],[15],[16]. APG has been described as a valuable tool to establish the relationships between arterial hypertension and its risk factors [17]; and the influencing factors of vascular compliance, including age and atherosclerosis, in a hypertensive [18].

Fig.4 shows the effect of auriculopuncture in the sympathetic point on the APG-b/a index in healthy subjects. The auriculopuncture at the sympathetic point elicited a significant increase in the APG-b/a index during the acupuncture period.

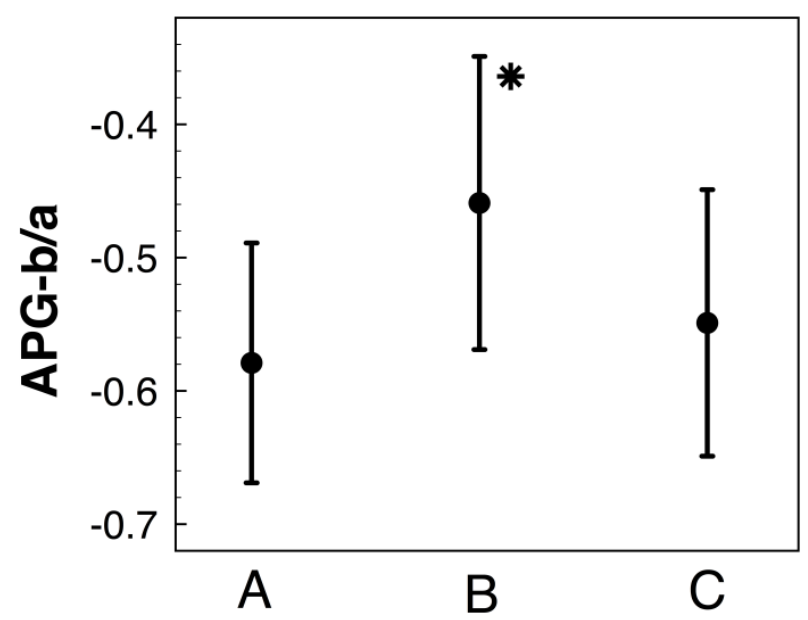

Fig.4. Effect of auriculopuncture in the sympathetic point on the APG-b/a index in healthy subjects

Fig.5 shows the effect of auriculopuncture in the sympathetic point on the APG-d/a index in healthy subjects. The auriculopuncture at the sympathetic point elicited a significant decrease in the APG-d/a index during the acupuncture period.

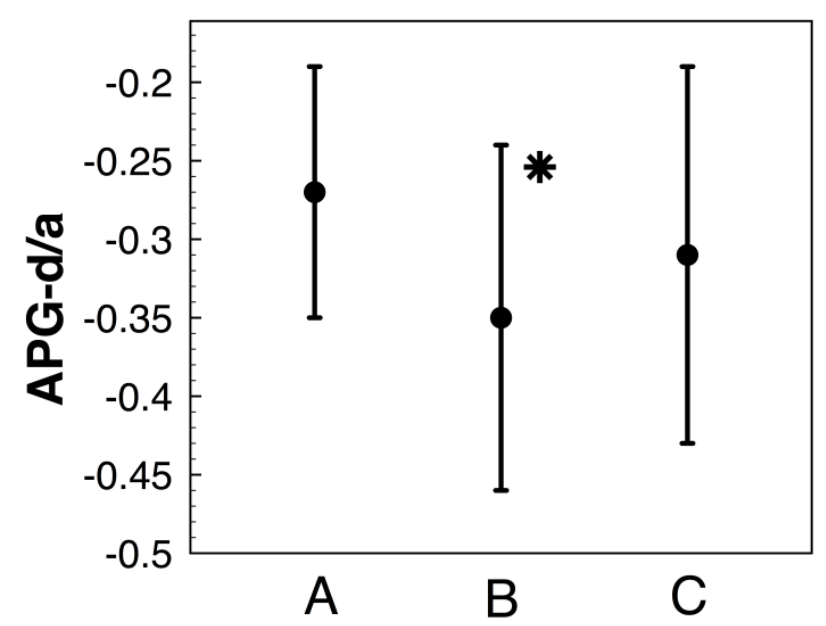

Fig.5. Acupuncture-induced changes in the acceleration photoplethysmogram index d/a (APG-d/a) of the right pinna sympathetic point. A: baseline period, B: acupuncture period, and C: after acupuncture period. Data are presented as mean \pm standard deviation. $* \mathrm{p}<0.05$, significantly different from baseline values, $\mathrm{p}<0.05$

Fig.6 shows the effect of auriculopuncture in the sympathetic point on the APG-AI index in healthy subjects. The auriculopuncture at the sympathetic point elicited a non-significant increase in the APG-AI index during the acupuncture period. 


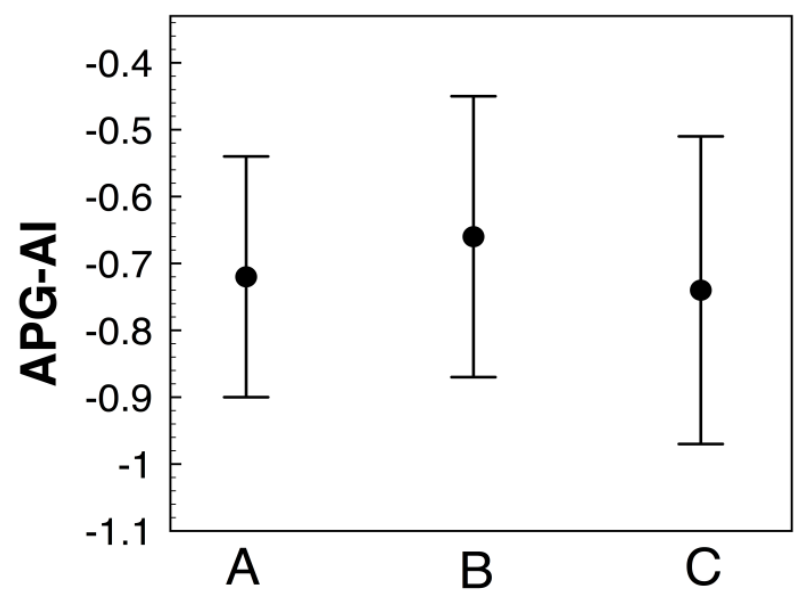

Fig.6. Acupuncture-induced changes in the acceleration photoplethysmogram aging index b/a (APG-AI) of the right pinna sympathetic point. A: baseline period, B: acupuncture period, and C: after acupuncture period. Data are presented as mean \pm standard deviation. $* \mathrm{p}<0.05$, significantly different from baseline values, $\mathrm{p}<0.05$

\section{Discussion}

Summary of results: The most important findings of the study were the following:

- Significant rise in the APG-ba index.

- Significant decrease in the APG-da index.

- Non-significant increase in the APG-AI index.

Interpretation of $\boldsymbol{A P G}$ indices: Various researchers have studied the physiological meanings and clinical implications of the APG waveform. It has been proposed that the b wave in the APG mainly expresses the first vascular response to the ejection of blood from the left ventricle and without interference from reflected distal components [9],[19],[20]. Besides, the a and b waves correspond to the early systolic component of the APG; therefore, the b/a ratio is considered as a marker of the compliance of the great arteries; consequently, b/a ratio is little affected by the reflection wave [18],[19].

Therefore, the present study showing the increase of APG-b/a index may indicate a decrease in arterial compliance. On the contrary, the d-wave corresponds to the late systolic component of the PPG, which is related to the reflected pulse, that is, the wave traveling backward from peripheral sites [18],[19]. The APG-d/a index is closely related to the late rise in systolic pressure in the ascending aorta. Therefore, it is considered a marker of the intensity of the wave reflection [18]. Besides, the $\mathrm{d}$ wave corresponds to the late systolic component of the PPG, which is related to the reflected pulse wave that travels retrograde [11]. Takazawa et al. [21] proposed that the APG-b/a index reflects high arterial stiffness and that the APG-d/a index helps assess vasoconstriction and the $\mathrm{d} / \mathrm{a}$ ratio decreases, the b/a ratio increases as a result of the application of vasoactive agents [21]. Besides, Imanaga et al. [12] reported the correlation between the b/a ratio and carotid artery compliance, suggesting that b/a reflects the stiffness of the great arteries. Otherwise, wave d mainly represents the intensity of the reflection wave. Therefore, Takazawa et al. [21] reported that the $d / a$ index was significantly correlated with the aortic enlargement index, partially suggesting a relationship with peripheral vascular resistance. 
The $\mathrm{d} / \mathrm{a}$ ratio and the late increase in the systolic pressure show a significant relationship in the ascending aorta arteriosclerosis; therefore, this index is considered a marker of the intensity of the wave reflection and can represent such vascular properties [21].

Auriculotherapy and the components of the photoplethysmogram: Several studies on the effect of auricular acupoints show diverse effects on sympathovagal balance. For example, acupuncture to specific ear points such as Shenmen significantly increases HRV [22]. Another study shows that electrical stimulation in the shell appears to be an appropriate method for stimulating the vagus nerve using auricular electrical stimulation in humans [23]. Otherwise, volunteers showed sympathomimetic effects when they underwent a randomized clinical trial with auricular acupuncture stimulation in treating obesity [24].

Also, the hypertensive groove auricular point modifies pulse transit time in healthy subjects [25]. Other results clearly showed the potential of the auricular acupuncture point of the Heart to modify $\mathrm{RI}_{\mathrm{DVP}}$ and $\mathrm{SI}_{\mathrm{DVP}}$; these indices are valuable for evaluating vascular tone and arterial stiffness [26].

Furthermore, auricular acupuncture points improve cardiovascular disease [27]. Auricular point stimulation is proposed to regulate cardiovascular function by activating baroreceptor-responsive neurons in the nucleus of the solitary tract [23].

\section{Conclusion}

Applying the sympathetic auriculopuncture point caused significant changes in the APG-b/a and APG-d/a indices. These changes are similar to those that originate with the administration of vasoconstrictors such as angiotensin II. The changes suggest variations in the anterograde and retrograde circulatory components of the arterial circulation.

\section{Declarations}

\section{Source of Funding}

This research did not receive any grant from funding agencies in the public, commercial, or not-for-profit sectors.

\section{Competing Interests Statement}

The authors declare no competing financial, professional and personal interests.

\section{Consent for publication}

Authors declare that they consented for the publication of this research work.

\section{Ethical Approval}

The Ethics Committee of the Biological and Health Sciences Division of the Autonomous Metropolitan University of Iztapalapa (2015.6.03.06) approved the trial. All participants gave informed consent.

\section{References}

[1] Mercante B, Ginatempo F, Manca A, Melis F, Enrico P, Deriu F. Anatomo-Physiologic Basis for Auricular Stimulation. Med Acupunct. 2018; 30(3): 141-150. 
[2] Rabischong P, Terral C. Scientific Basis of Auriculotherapy: State of the Art. Med Acu. 2014; 26(2): 84-96.

[3] Yeh ML, Chang YC, Huang YY, Lee TY. A randomized controlled trial of auricular acupressure in heart rate variability and quality of life for hypertension. Complement Ther Med. 2015; 23(2): 200-9.

[4] Gao XY, Li YH, Liu K, Rong PJ, Ben H, Li L, Zhu B, Zhang SP. Acupuncture-like stimulation at auricular point Heart evokes cardiovascular inhibition via activating the cardiac-related neurons in the nucleus tractus solitarius. Brain Res. 2011; 1397: 19-27.

[5] Allen J. Photoplethysmography and its application in clinical physiological measurement. Physiol Meas. 2007 Mar; 28(3): R1-39.

[6] Nichols WW. Clinical measurement of arterial stiffness obtained from noninvasive pressure waveforms. Am J Hypertens. 2005; 18(1 Pt 2): 3S-10S.

[7] López-Beltrán EA, Blackshear PL, Finkelstein SM, Cohn JN. Non-invasive studies of peripheral vascular compliance using a non-occluding photoplethysmographic method. Med Biol Eng Comput. 1998; 36(6): 748-53.

[8] Elgendi M, Fletcher R, Liang Y, Howard N, Lovell NH, Abbott D, Lim K, Ward R. The use of photoplethysmography for assessing hypertension. NPJ Digit Med. 2019 Jun 26; 2: 60.

[9] Takada Imanaga I, Hara H, Koyanagi S, Tanaka K. Correlation between wave components of the second derivative of plethysmogram and arterial distensibility. Jpn Heart J. 1998; 39(6): 775-84.

[10] Fleischhauer V, Ruprecht N, Sorelli M, Bocchi L, Zaunseder S. Pulse decomposition analysis in photoplethysmography imaging. Physiol Meas. 2020; 41(9): 095009.

[11] Takada H, Washino K, Harrell JS, Iwata H. Acceleration plethysmography to evaluate aging effect in cardiovascular system. Using new criteria of four wave patterns. Med Prog Technol. 1996-1997; 21(4): 205-10.

[12] Imanaga I, Hara H, Koyanagi S, Tanaka K. Correlation between wave components of the second derivative of plethysmogram and arterial distensibility. Jpn Heart J. 1998; 39(6): 775-84.

[13] Elgendi M. Standard terminologies for photoplethysmogram signals. Curr Cardiol Rev. 2012; 8(3): 215-9.

[14] Elgendi M. Detection of c, d, and e waves in the acceleration photoplethysmogram. Comput Methods Programs Biomed. 2014; 117(2): 125-36.

[15] Savitzky A, Golay MJE. Smoothing and differentiation of data by simplified least squares procedures. Ann Chem 1964; 36: 1627-39.

[16] Ahn JM. New aging index using signal features of both photoplethysmograms and acceleration plethysmograms. Healthc Inform Res. 2017; 23(1): 53-59.

[17] Hashimoto J, Chonan K, Aoki Y, Nishimura T, Ohkubo T, Hozawa A, Suzuki M, Matsubara M, Michimata M, Araki T, Imai Y. Pulse wave velocity and the second derivative of the finger photoplethysmogram in treated hypertensive patients: their relationship and associating factors. J Hypertens. 2002; 20(12): 2415-22. 
[18] Bortolotto LA, Blacher J, Kondo T, Takazawa K, Safar ME. Assessment of vascular aging and atherosclerosis in hypertensive subjects: second derivative of photoplethysmogram versus pulse wave velocity. Am J Hypertens. 2000; 13(2): 165-71.

[19] Hashimoto J, Watabe D, Kimura A, Takahashi H, Ohkubo T, Totsune K, Imai Y. Determinants of the second derivative of the finger photoplethysmogram and brachial-ankle pulse-wave velocity: the Ohasama study. Am J Hypertens. 2005; 18(4 Pt 1): 477-85.

[20] Elgendi M. On the analysis of fingertip photoplethysmogram signals. Curr Cardiol Rev. 2012;8(1):14-25.

[21] Takazawa K, Tanaka N, Fujita M, Matsuoka O, Saiki T, Aikawa M, Tamura S, Ibukiyama C. Assessment of vasoactive agents and vascular aging by the second derivative of photoplethysmogram waveform. Hypertension. 1998; 32(2): 365-70.

[22] Wang L, Cheng W, Sun Z, Xu Y, Cheng G, Gaischek I, Kuang H, Litscher G. Ear acupressure, heart rate, and heart rate variability in patients with insomnia. Evid Based Complement Alternat Med. 2013; 2013: 763631.

[23] Gao XY, Li YH, Liu K, Rong PJ, Ben H, Li L, Zhu B, Zhang SP. Acupuncture-like stimulation at auricular point Heart evokes cardiovascular inhibition via activating the cardiac-related neurons in the nucleus tractus solitarius. Brain Res. 2011; 1397: 19-27.

[24] Shen EY, Hsieh CL, Chang YH, Lin JG. Observation of sympathomimetic effect of ear acupuncture stimulation for body weight reduction. Am J Chin Med. 2009; 37(6): 1023-30.

[25] Rivas-Vilchis JF, Moisés Salvador Castañeda-Ramírez MS. The hypertension groove auricular acupoint modifies pulse transit time in healthy subjects. Rev Int Acupuntura 2019; 13: 89-95.

[26] Serrano-Morales MM, Mejía-González MM1, moisés salvador castañeda-ramírez MS2, josé federico Rivas-Vilchis JF. Effect of acupuncture in heart auricular point in two indexes of digital volume pulse in healthy subjects. Asian J Pharm Clin Res. 2020; 13: 5-7.

[27] Huang H, Liang S. Acupuncture at otoacupoint heart for treatment of vascular hypertension. J Tradit Chin Med. 1992; 12(2): 133-6. 